\title{
Lattice QCD calculation of direct CP violation and long distance effects in kaon mixing and rare decays
}

\author{
Norman Christ* \\ Department of Physics, Columbia University, New York, NY 10027, USA \\ E-mail: nhcephys.columbia.edu
}

\section{RBC and UKQCD Collaborations}

With the recently achieved ability to work directly at the physical pion mass, a variety of important quantities become accessible to lattice calculation. We will discuss recent results and future prospects for the first-principles calculation of $\varepsilon^{\prime}$, the $K_{L^{-}} K_{S}$ mass difference and the long distance contribution to other rare weak processes such as $\varepsilon, K^{+} \rightarrow \pi^{+} v \bar{v}$ and $K_{S} \rightarrow \pi^{0} \ell^{+} \ell^{-}$.

Flavor Physics \& CP Violation 2015

May 25-29, 2015

Nagoya, Japan

\footnotetext{
* Speaker.
} 


\section{Introduction}

With the recent availability of petaflops-scale computers and the continued, impressive advances in numerical algorithms, lattice field theory methods can now be used to calculate the predictions of the standard model for an increasing number of processes for which there are reasonable expectations for the discovery of new physics. This is true both for phenomena which are dominated by the non-perturbative behavior of QCD such as direct CP violation in $K \rightarrow \pi \pi$ decay and $\Delta M_{K}=M_{K_{L}}-M_{K_{S}}$ but also for those in which accurate, perturbative treatment is possible but where small, non-perturbative QCD effects would otherwise limit the sensitivity to new physics.

In this talk we will discuss four processes in which the methods of lattice QCD are beginning or promising to play an important role in the comparison between experimental results and the predictions of the standard model. For the case of direct CP violation in $K \rightarrow \pi \pi$ decays and the parameter $\varepsilon^{\prime}$ there are now first results [U] from a calculation at a single lattice spacing with physical kinematics and estimated errors, expressed as a fraction of the experimental result, that are on the order of $30 \%$. For the other three topics, $\Delta M_{K}$ and the long distance corrections to the standard model predictions for the indirect CP violation parameter $\varepsilon$ and the rare kaon decay $K^{+} \rightarrow \pi^{+} v \bar{v}$, we present exploratory studies in which we demonstrate the feasibility of the proposed calculations but where physical results await lattice QCD ensembles with the small lattice spacing that is needed to properly treat the relatively massive charm quark.

\section{2. $K \rightarrow \pi \pi$ decay and direct $\mathrm{CP}$ violation}

Calculating the parameter $\varepsilon^{\prime}$ which describes the direct CP violation present in the two-pion decay of the kaon has been a long-term goal of the RBC and UKQCD collaborations for many years and an actual 3-flavor calculation with physical quark masses and pion momenta has been underway for four years. During the conference we described the status of this calculation and showed preliminary results for lattice matrix elements. After the meeting, first results from this calculation have been presented [W] and it is those results that will be described here.

The first step in such a lattice calculation is the use of QCD and electroweak perturbation theory to represent the standard model by an effective four-fermion theory. While the weak decay amplitudes show structure at the $100 \mathrm{GeV}$ energy scale of the $W$ and $Z$ boson masses, at the much lower, $1 \mathrm{GeV}$ energies present in $K$ meson decay the relevant weak decay amplitudes show no electro-weak structure and are accurately represented by the local effective Hamiltonian density

$$
\mathscr{H}^{\Delta S=1}=\frac{G_{F}}{\sqrt{2}} V_{u d} V_{u s}^{*}\left\{\sum_{i=1}^{10}\left[z_{i}(\mu)+\tau y_{i}(\mu)\right] Q_{i}\right\} .
$$

Here the $Q_{i}$ are ten four-fermion operators (of which seven are independent) that are specified in Ref. [2] in terms of the conventions we use. The quantities $V_{q^{\prime} q}$ are the CKM matrix elements connecting the charge $-1 / 3$ quark $q$ and the charge $+2 / 3$ quark $q^{\prime}$ while the ratio $\tau=$ $-\left(V_{t d} V_{t s}^{*}\right) /\left(V_{u d} V_{u s}^{*}\right)$ carries the $C P$-violating phase that leads to a non-zero value of $\varepsilon^{\prime}$. The fourfermion operators $Q_{i}$ may be best described by giving an example. The "gluonic penguin" operator $Q_{6}$ which makes the largest contribution to $\varepsilon^{\prime}$ is given by

$$
Q_{6}=\sum_{q=u, d, s} \bar{s}_{a} \gamma^{v}\left(1-\gamma^{5}\right) d_{b} \bar{q}_{b} \gamma^{\nu}\left(1+\gamma^{5}\right) q_{a}
$$


where $u, d$ and $s$ are the field operators for the up, down and strange quarks and the vector index $v$ and color indices $a$ and $b$ are summed over.

The decay of a neutral kaon into two pions can be described in terms of three amplitudes [B] . The first two are the complex amplitudes, $A_{0}$ and $A_{2}$ which describe the matrix elements of $\mathscr{H}^{\Delta S=1}$ between the neutral kaon with negative strangeness $\left(K^{0}\right)$, and a two-pion state with isospin $I=0$ or 2:

$$
A_{I} e^{i \delta_{I}}=\left\langle\pi \pi(I)\left|\mathscr{H}^{\Delta S=1}\right| K^{0}\right\rangle \quad \text { for } \quad I=0,1,
$$

where $\delta_{I}$ is the $\pi \pi$ scattering phase shift for $I=0$ or 2 . The third is the complex parameter $\bar{\varepsilon}$ which specifies the mixtures of $K^{0}$ and $\bar{K}^{0}$ which make up the long- and short-lived decay eigenstates $K_{L}$ and $K_{S}$ :

$$
K_{S}=\frac{K_{2}^{0}+\bar{\varepsilon} K_{1}^{0}}{\sqrt{1+|\varepsilon|^{2}}} \quad K_{L}=\frac{K_{1}^{0}+\bar{\varepsilon} K_{2}^{0}}{\sqrt{1+|\varepsilon|^{2}}},
$$

where $K_{1}$ and $K_{2}$ are the $\mathrm{CP}$ even and odd eigenstates, $\left(K^{0} \mp \bar{K}^{0}\right) / \sqrt{2}$. The two conventional parameters $\varepsilon$ and $\varepsilon^{\prime}$ which describe indirect and direct CP violation in $K \rightarrow \pi \pi$ decay and can be measured directly in experiment are related to these three quantities by the formulae:

$$
\begin{aligned}
\varepsilon & =\bar{\varepsilon}+i \frac{\operatorname{Im} A_{0}}{\operatorname{Re} A_{0}} \\
\varepsilon^{\prime} & =\frac{i e^{i\left(\delta_{2}-\delta_{0}\right)}}{\sqrt{2}}\left|\frac{A_{2}}{A_{0}}\right|\left(\frac{\operatorname{Im} A_{2}}{\operatorname{Re} A_{2}}-\frac{\operatorname{Im} A_{0}}{\operatorname{Re} A_{0}}\right) .
\end{aligned}
$$

In the standard model, the indirect $\mathrm{CP}$ violation parameter $\varepsilon$ is dominated by amplitudes which contain a top quark and hence by short distances. As a result $\varepsilon$ can be determined by the product a Wilson coefficient determined by short-distance electro-weak perturbation theory and the $K^{0}-\bar{K}^{0}$ matrix element of a local operator that is calculated accurately using lattice QCD. The long distance corrections to this picture, expected to be at or below the 5\% level, are the subject of Sec. 因.

As can be seen from Eq. (ㅁ. $)$, the parameter $\varepsilon^{\prime}$ characterizing direct CP violation in $K^{0}$ decay results from a relative phase between the amplitudes $A_{0}$ and $A_{2}$, both determined by matrix elements which are natural targets for lattice QCD calculation. In fact, the amplitude complex $A_{2}$ was first computed in lattice QCD five years ago [䧃] and is now determined in the continuum limit [可] with an accuracy of $12 \%$. The lattice calculation of $A_{0}$ is substantially more difficult because of the presence of "disconnected diagrams" and the challenge of studying an $I=0 \pi \pi$ state in which the pions carry a relative momentum of $205 \mathrm{MeV}$.

For both the earlier calculation of $A_{2}$ and the present calculation of $A_{0}$ we rely on the method developed by Lellouch and Lüscher [目] in which the finite volume of a lattice calculation is exploited to quantize the $\pi \pi$ energy, leading to a series of energy eigenstates, one of which can have an energy tuned to match that of the mass $M_{K}$ of the decaying $K$ meson. (A necessary finite volume correction is also provided as part of the Lellouch-Luüscher method.) In both our $A_{2}$ and $A_{0}$ calculations we carry this approach a step farther and adopt boundary conditions so that the least energetic, finite-volume, $\pi \pi$ state has an energy equal to $M_{K}$. For the $I=2$ case the $\pi \pi$ state is the lightest $I=2$ state in QCD so the large time limit of the standard Euclidean Green's function then projects onto the physical final $\pi \pi$ state:

$$
\left\langle O_{\pi \pi}^{I=2}\left(t_{\pi \pi}\right) \mathscr{H}^{\Delta S=1}\left(t_{\mathrm{op}}\right) O_{K}\left(t_{K}\right)\right\rangle \asymp N_{\pi \pi} N_{K} e^{-\left(t_{\pi \pi}-t_{\mathrm{op}}\right) E_{\pi \pi}} e^{-\left(t_{\mathrm{op}}-t_{K}\right) M_{K}}\left\langle\pi \pi^{I=2}\left|\mathscr{H}^{\Delta S=1}\right| K\right\rangle .
$$


Here $N_{\pi \pi}^{I=2}$ and $N_{K}$ are normalization factors for the $\pi \pi$ and kaon interpolating operators $O_{\pi \pi}^{I=2}$ and $O_{K}$ and the time differences $t_{\pi \pi}-t_{\mathrm{op}}$ and $t_{\mathrm{op}}-t_{K}$ are assumed to be sufficiently large that the contribution from other possible intermediate states can be neglected. Thus, for the calculation of $A_{2}$ the desired matrix element appears in the term which dominates the large time limit.

For the $I=0$ state the leading term in the large-time limit is the vacuum, the only $I=0$ state with a lower energy than the $E_{\pi \pi}=M_{K}, I=0$, finite-volume $\pi \pi$ state. While this vacuum contribution to the $I=0$ analogue of Eq. (2.7) can be very accurately determined and subtracted, the remaining term of interest, suffers from large stochastic noise. This is the problem of "disconnected" diagrams in QCD. The quantum correlations between the quantities $O_{\pi \pi}^{I=0}\left(t_{\pi \pi}\right)$ and $\mathscr{H}^{\Delta S=1}\left(t_{\mathrm{op}}\right)$ at large separation do not result from an explicit quark propagator joining these two quantities since there is no quark line "connecting" these two observables. Instead we must rely on the exponentially falling correlations between fluctuations which are of order one - a situation which leads to an exponentially falling signal-to-noise ratio.

This disconnected diagram problem is reduced to a manageable level by collecting many Monte Carlo samples and choosing $O_{\pi \pi}^{I=0}\left(t_{\pi \pi}\right)$ in such a way as to minimize the contribution of the vacuum state. In particular, $O_{\pi \pi}^{I=0}\left(t_{\pi \pi}\right)$ is constructed as a product of two single-pion operators introduced at the separated times $t_{\pi \pi}$ and $t_{\pi \pi}+4$. These two single-pion operators are themselves formed from quark and anti-quark creation operators whose relative coordinate $r$ is distributed as $e^{-2 r}$ where $r$ is given in lattice units. Each correlated, quark-anti-quark pair, is uniformly distributed over the entire time slice at $t_{\pi \pi}$ or $t_{\pi \pi}+4$. Both the separation of the pions in time and the use of a tightly localized pion source each reduce the error $2 \times$, equivalent to a $16 \times$ increase in statistics.

Choosing boundary conditions to remove finite-volume $\pi \pi$ states with energy below $M_{K}$ is easiest for the $I=2$ case. Here isospin symmetry can be used to relate the Green's function needed to determine $A_{2}$ to one containing a isospin-transformed $\mathscr{H}^{\Delta S=1}$ operator and a $\pi^{+} \pi^{+}$interpolating operator. Since this there is no $I=0$ state with two units of charge with which the $\pi^{+} \pi^{+}$state can mix, we can impose boundary conditions which violate isospin symmetry without introducing unwanted finite-volume mixing. Assigning periodic boundary conditions to the up quark and antiperiodic boundary conditions to the $d$ quark in all three directions is sufficient for our two, largest, $5.3 \mathrm{fm}$, lattice volumes to ensure that the lowest energy $\pi \pi$ state has energy equal to $M_{K}$, once the effects of $I=2 \pi \pi$ repulsion are taken into account.

The same effect can be achieved for the $I=0$ case if G-parity boundary conditions are imposed $[$ []]. Recall that G-parity is a symmetry of QCD which combines a charge conjugation operation with a isospin rotation through $\pi$ about the $y$ axis. Thus, the usual doublet of $u$ and $d$ fields transforms under G-parity as:

$$
\left(\begin{array}{l}
u \\
d
\end{array}\right) \quad \stackrel{G}{\longrightarrow}\left(\begin{array}{c}
-C \bar{d}^{T} \\
C \bar{u}^{T}
\end{array}\right)
$$

where $u, d, \bar{u}$ and $\bar{d}$ can be viewed as the 4-component spinors formed of Grassmann, pathintegration variables and $C$ the usual $4 \times 4$ matrix that appears in the charge conjugation operation. G-parity boundary conditions require that when the Dirac operator connects fermion fields on either side of a spatial boundary, a G-parity transformation in interposed. This has the consequence 
that the QCD gauge links which cross this boundary join $\mathrm{SU}(3)$ ' 3 ' and ' 3 ' representations rather than ' 3 ' and ' $\overline{3}$ ' representations. Thus, these links transform differently under gauge transformation which means that the plaquettes that cross the boundary must also be special. Consequently, calculations that involve valence quarks which obey G-parity boundary conditions must be performed on an ensemble that has been generated using a special gauge action satisfying chargeconjugation boundary conditions and any fermion action used with this ensemble must involve charge-conjugation or G-parity boundary conditions.

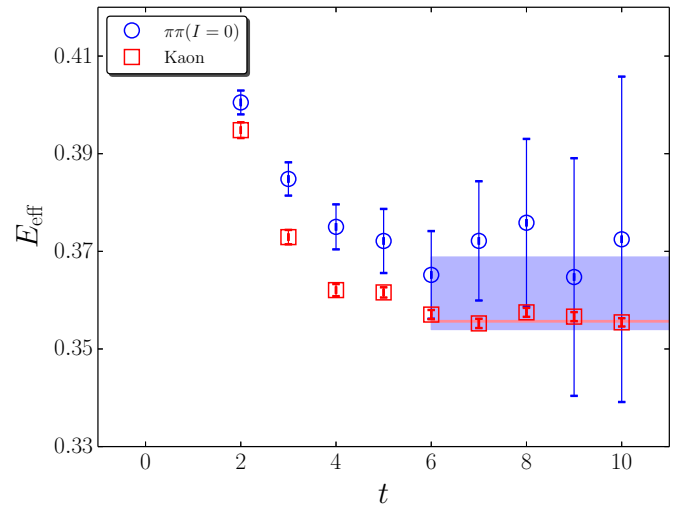

Figure 1: The effective mass of the kaon and twopion states obtained from the kaon and two-pion two-point Green's functions. The shaded bands show the $\pm 1 \sigma$ range of our results and $M_{K} \approx E_{\pi \pi}$.

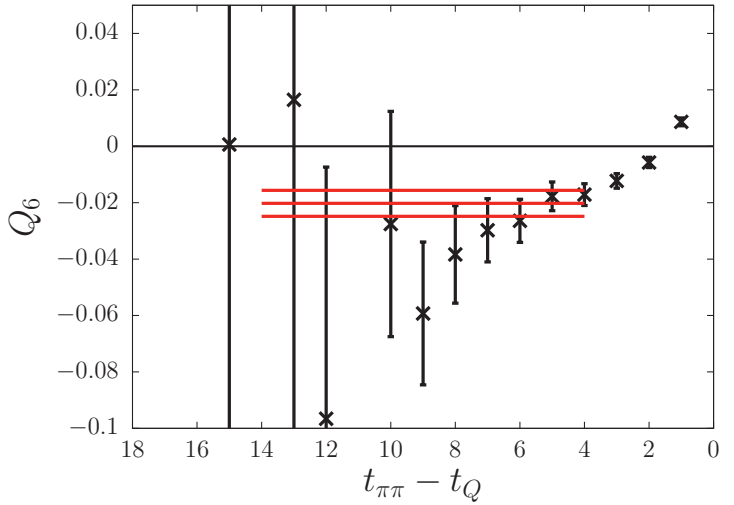

Figure 2: Results for the matrix element of $Q_{6} \mathrm{ob}-$ tained from a variant of Eq. (2.7) in which the operator $\mathscr{H}^{\Delta S=1}\left(t_{\mathrm{op}}\right)$ is replaced by $Q_{6}\left(t_{Q}\right)$ plotted versus the minimum $Q_{6}-O_{\pi \pi}$ time separation.

We now have obtained first results for $A_{0}$ using physical kinematics, a $4.6 \mathrm{fm}$ box and an inverse lattice spacing of $1 / a=1.38 \mathrm{GeV}$. Figures $\square$ and $\square$ from Ref. [W] show two important components of this calculation. The left-hand plot shows the effective mass of the kaon and two-pion states obtained from the time dependence of the kaon and two-pion, two-point functions. The plotted mass is obtained by comparing the expected hyperbolic cosine, single-state time dependence with the ratio of the two-point function at the plotted times $t$ and $t+1$. Our result for the finite-volume, $I=0$ two-pion energy, $E_{\pi \pi}=498(11) \mathrm{MeV}$ is obtained from a single-state fit to the time-dependence of the $\pi \pi$ two-point function including source-sink time separations no smaller than 6. This energy can be combined with Lüscher's quantization condition [ $[\mathbb{8}, \mathbf{Q}]$ to obtain the two-pion phase shift and gives $\delta_{0}=23.8(4.9)(1.2)^{\circ}$ at this value of $E_{\pi \pi}$, significantly below the expectations of phenomenology for the $\pi \pi$ energy. Aware of this discrepancy, we carefully examined our determination of $E_{\pi \pi}$ finding consistent results from single and two-state fits.

Figure $\square$ shows the result for the matrix element of the important QCD-penguin operator $Q_{6}$ between kaon and $\pi \pi$ states. The result plotted for a specific value of $t_{\pi \pi}-t_{Q}$ is obtained from all Green's function results with at least that value for the time separation between the $O_{\pi \pi}$ and $Q_{6}$ operators and a time separation of at least 6 six lattice units between the kaon and $Q_{6}$ operators. For the ten weak operators which enter the determination of $A_{0}$ we use a minimum $O_{\pi \pi^{-}} Q_{i}$ time separation of four lattice units. Using Eq. (ㄷ.6) and experimental values for $\operatorname{Re}\left(A_{0}\right)$ and $\operatorname{Re}\left(A_{2}\right)$ we obtain

$$
\operatorname{Re}\left(\varepsilon^{\prime} / \varepsilon\right)=1.38(5.15)(4.59) \times 10^{-4}
$$


$2.1 \sigma$ below the experimental value of $16.6(2.3) \times 10^{-4}$. In this determination we do not use our lattice value for $\operatorname{Re}\left(A_{0}\right)$ in order to increase the accuracy of our result for $\varepsilon^{\prime}$. However, our result for $\operatorname{Re}\left(A_{0}\right), 4.66(1.00)(1.26) \times 10^{-7} \mathrm{GeV}$, agrees well with the experimental value of $3.3201(18) \times$ $10^{-7} \mathrm{GeV}$.

While the agreement between this first-principles, standard model result and the experimental value for $\varepsilon^{\prime}$ is already of considerable interest [ए]] , much can be done to increase the precision of the current lattice calculation. Over the next two years we expect to be able to continue the current calculation, quadrupling the present Monte Carlo statistics and substantially reducing renormalization errors associated with representing the effective weak interaction theory by lattice operators. In the longer term, calculations with new ensembles with multiple values for the lattice spacing will allow a continuum limit to be evaluated leading to total errors at the $10 \%$ scale. Also important is the development of alternative lattice methods to compute $\varepsilon^{\prime}$ and Ref. [W] is a good step in this direction.

\section{3. $K_{L}-K_{S}$ mass difference}

While less mature than the $K \rightarrow \pi \pi$ calculations described above, much progress has been made toward a physical, first-principles lattice QCD calculation of $\Delta M_{K}$. In the standard model, the $K_{L}-K_{S}$ mass difference results from a second order weak process which is dominated by energies at or below the scale of the charm quark mass. Thus, in contrast with the $K \rightarrow \pi \pi$ calculation, two effective weak Hamiltonians are involved, potentially separated by distances of the charm quark Compton wavelength or larger. Such a second-order quantity can be obtained from the usual integrated, time-order product of the factors of $\mathscr{H}^{\Delta S=1}$ and/or $\mathscr{H}^{\Delta S, C=1}$. However, in a Euclidean lattice calculation additional unwanted terms appear which grow exponentially in the necessary large time limit [12]. These exponentially growing terms come from states that are less massive than the kaon so, as a result, a calculation of $\Delta M_{K}$ is potentially more difficult than the above calculation of $K \rightarrow \pi \pi$ since now exponentially larger contributions from not only the vacuum but also one or two intermediate pions or even an intermediate $\eta$ must be removed and the correspondingly larger noise overcome.

In practice, this calculation appears less difficult than anticipated. A complete calculation with all diagrams, including disconnected and double-penguin graphs, has been performed using a 330 $\mathrm{MeV}$ pion mass on a $2.7 \mathrm{fm}, 24^{3} \times 64$ lattice volume [[3]] obtaining a result with $13 \%$ statistical errors from 500 gauge configurations. This calculation has been extended to $170 \mathrm{MeV}$ pions and a $(4.6 \mathrm{fm})^{3}$ spatial volume, also with promising results [14]]. These lighter pions result in a greater enhancement for the unwanted terms and the first appearance of unwanted two-pion states and their potentially important, finite-volume effects [니]. In each case the new effects were found to be small and manageable.

The greatest difficulty that must be overcome in a calculation of $\Delta M_{K}$, as well as those discussed in the two sections below, is the necessity of working with physically light pions in a calculation that also includes charm quarks and must therefore use a corresponding small lattice spacing. The series of very fine ensembles based on chiral fermions needed to properly treat the charm quark and evaluate the continuum limit may be 3-4 years away. As a first step we are now planning to mount a realistic calculation of $\Delta M_{K}$ using an existing, domain wall fermion, $64^{3} \times 128, L=5.3$ 
fm ensemble with $1 / a=2.38 \mathrm{GeV}$. While this will not allow a continuum limit, we expect that the finite lattice spacing errors associated with the charm quark will be at or below the $20 \%$ level. This planned calculation of $\Delta M_{K}$, which properly incorporates long-distance effects, is expected to be significantly more accurate than the impressive, existing NNLO perturbative calculation [ए6] in which the NNLO correction is $36 \%$ of the LO and NLO result and long-distance effects must be incorporated perturbatively.

\section{Long distance contribution to $\varepsilon$}

Closely related to the above calculation of $\Delta M_{K}$ is the use of lattice methods to determine the long-distance component of the indirect $\mathrm{CP}$ violation parameter $\varepsilon$. In contrast to $\Delta M_{K}$ the $\mathrm{CP}$ violating portion of $K^{0}-\bar{K}^{0}$ mixing comes mostly from short distances and can be expressed as a standard short-distance Wilson coefficient multiplying a four-quark operator whose matrix element determines the standard "bag parameter" $B_{K}$. Here the long distance effects are expected to be at or below the $5 \%$ level. However, they are now of considerable importance since $\varepsilon$ is known experimentally to $0.5 \%$ precision and lattice calculations of $B_{K}$ are approaching $1 \%$ accuracy.

While the calculation of the long-distance component of $\varepsilon$ shares much with the calculation of $\Delta M_{K}$ there is an important new challenge. Because $\varepsilon$ is short-distance dominated the lattice calculation is not self-contained. If $\Delta M_{K}$ is computed in four-flavor lattice QCD using the standard, effective, four-flavor weak Hamiltonian the calculation is finite once the four-quark operators have been properly normalized. However, in the corresponding four-flavor, effective field theory calculation of $\varepsilon$ contains a new ultraviolet, logarithmic divergence associated with the collision of the locations of the two four-quark operators. In a continuum theory this divergence would be regulated by the short-distance structure of the weak interactions. This structure has been omitted from the low-energy effective theory and under any circumstances would be inaccessible to a lattice calculation with a lattice cutoff of $1 / a=2-3 \mathrm{GeV}$.

The appearance of new divergences when an effective low-energy theory is used at second order is entirely standard and new, low-energy constants must be introduced to replace the divergent quantities. These new low-energy constants must be chosen to correctly describe the physical, highenergy theory and must be provided to make the low-energy description complete. For the case of $\varepsilon$ the single, new low-energy constant represents the dominant, high-energy contribution to $\varepsilon$, a quantity accurately captured by the usual short-distance calculation of $\varepsilon$.

The Rome-Southampton renormalization technique provides a familiar and effective way to combine the lattice calculation (with its short-distance ambiguity) and the continuum, short-distance calculation (with its failure to correctly describe the long-distance non-perturbative regime) [ए2]. In this approach we identify an off-shell, Landau-gauge-fixed, infrared save, $\Delta S=2$ Green's function, evaluated at a momentum scale $\mu$. We choose $\mu \gg \Lambda_{\mathrm{QCD}}$ to ensure that this Green's function can be computed in perturbation theory. We also require that $\mu \ll 1 / a$ so that a lattice calculation in the effective theory will also be accurate. Finally we include the $\Delta S=2$ four-quark operator required for the effective theory to be applicable in second order and fix its coefficient by requiring that this Green's function, evaluated at the scale $\mu$ take the correct standard model value computed in perturbation theory. 
A pilot calculation of the sort outlined above has been carried out on a $24^{3} \times 64$ ensemble with unphysical quark masses [ㅁ]]. The results have an accuracy similar to that found in the calculation of $\Delta M_{K}$. At least in this example, the Rome-Southampton treatment of the needed low-energy constant also works as expected, with the final result for $\varepsilon$ showing only a small dependence on the arbitrary renormalization scale $\mu$. As in the case of the calculation of $\Delta M_{K}$, a first-principles calculation of the long-distance component of $\varepsilon$ in the standard model is well within the reach of lattice QCD but requires sufficiently large volumes and fine lattice spacings to allow the simultaneous accurate treatment of both light physical pions and heavy physical charm quarks.

\section{Long distance contribution to rare kaon decays}

The rare kaon decays, $K \rightarrow \pi \ell \bar{\ell}$ and $K \rightarrow \pi v \bar{v}$ have long been an important focus for experimental and theoretical research. As second-order, highly suppressed processes in the standard model, they provide important opportunities for the discovery of phenomena which lie beyond the standard model. These rare decays provide favored standard model tests because in important cases theoretically clean standard model predictions can be made which are unaffected by the non-perturbative uncertainties of QCD. However, as first proposed by Isodori et al. [ए8], lattice calculations can be used to increase the precision or remove ambiguities of these predictions.

These issues have been studied recently in detail by the RBC and UKQCD collaborations and the $K \rightarrow \pi \ell \bar{\ell}$ decays are discussed in Ref. [प्प] and are now the object of exploratory calculations. Of special interest is the possibility of determining the unknown sign of indirect CP violation in the decay $K_{L} \rightarrow \pi^{0} \ell \bar{\ell}$. Here we consider the $K^{+} \rightarrow \pi^{+} v \bar{v}$ decay which is the focus of the NA62 CERN experiment and where long distance effects, although small $(\approx 4 \%)$, are the leading theoretical uncertainty. The two types of diagram which contain relevant long-distance effects, are shown in Fig. B]. Of particular interest is the lower, $W$-exchange diagram which contains an internal lepton propagator.

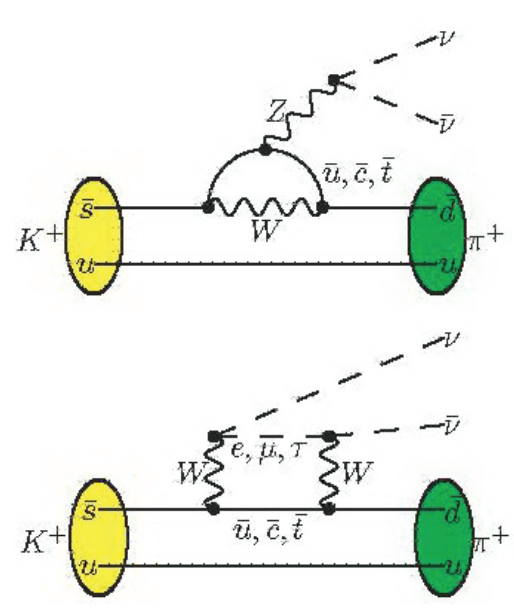

Figure 3: Examples of the two types of diagrams that contribute to the rare kaon decay $K^{+} \rightarrow \pi^{+} v \bar{v}$
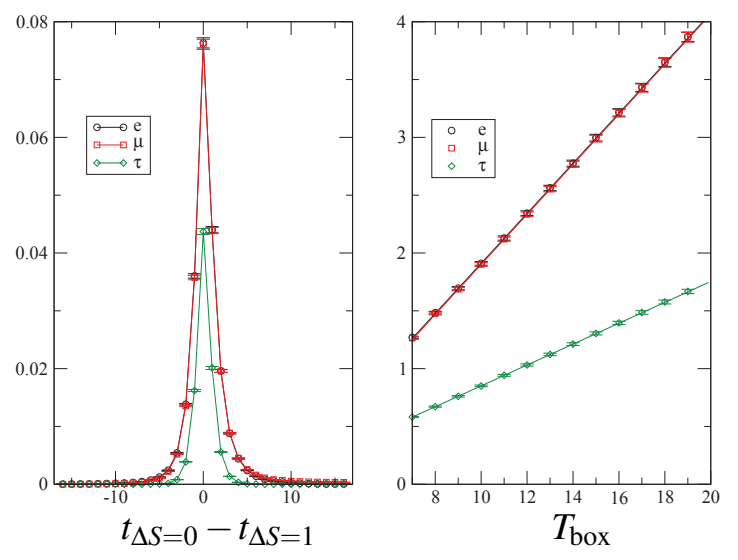

Figure 4: Results for the lower diagram in Fig. 3 as explained in the text. The slope of the lines in the right-hand panel determine the matrix element.

Here also detailed exploratory calculations are underway on both $16^{3} \times 32$ and $32^{3} \times 64$ lattice

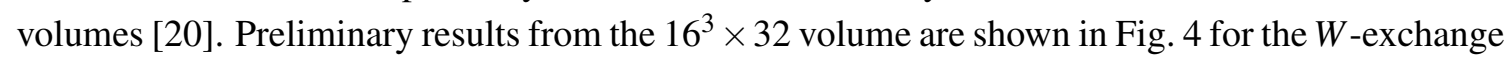


diagram in the lower part of Fig. [1. The left panel of Fig. 田 shows the result as a function of $t_{\Delta S=0}-t_{\Delta S=1}$ for the unintegrated correlator in which the $\Delta S=1$ two-quark/two-lepton vertex is integrated over the time slice at the time $t_{\Delta S=1}$ while the $\Delta S=0$ two-quark/two-lepton vertex is integrated over the time slice at the time $t_{\Delta S=0}$. The right hand panel shows the result when these two times are integrated over an interval of length $T_{\text {box }}$. The slopes of the lines seen in the righthand panel of Fig. 团 determine the decay matrix element for the particular momenta assigned to the initial $K^{+}$and final $\pi^{+}, v$ and $\bar{v}$. In each figure the three curves correspond to the three types of intermediate charged lepton and corresponding final-state neutrinos.

As can be seen, statistical errors on the order of $2 \%$ are obtained for this type of diagram. Disconnected diagrams in which the $Z$ boson in the upper panel of Fig. B attach to an isolated quark loop have much larger relative statistical errors but the small absolute size of these terms implies that a complete calculation of all amplitudes is practical. As in the case of the long distance component of $\varepsilon$ discussed earlier, this calculation must be performed in the four-flavor theory. As in that case, even with the resulting up-charm "GIM" cancelation, the amplitude contains a logarithmic short-distance singularity so that a new low-energy constant must be added to the effective theory. As in the case of the $\varepsilon$ calculation, this low-energy constant can be fixed using Rome-Southampton methods and electro-weak/QCD perturbation theory.

\section{Conclusion}

The methods and resources available for lattice QCD are making possible the first-principles calculation of an increasing array of important physical quantities. At present these calculations are performed using the standard model and allow increasingly accurate and varied comparisons between the standard model and experiment. Many of the quantities that are believed to be sensitive to new, beyond-the-standard-model phenomena, some measured precisely decades ago, will soon provide substantially more challenging tests of the standard model. In fact, it is becoming increasingly timely to ask when will these rapidly improving lattice results justify new, higher-precision experiments? With all of the workshop participants, we look forward to the day when the methods at hand, here those of lattice $\mathrm{QCD}$, are being used to characterize new theories intended to explain observed discrepancies between experiment and the standard model.

I would like to thank my RBC and UKQCD collaborators whose work is reported here and acknowledge support from the U.S. DOE grant, DE-SC0011941.

\section{References}

[1] RBC, UKQCD Collaboration, Z. Bai et al., Standard Model Prediction for Direct CP Violation in $K \rightarrow \pi \pi$ Decay, Phys. Rev. Lett. 115 (2015), no. 21212001 [arXiv:1505.07863 [hep-lat]].

[2] RBC Collaboration, T. Blum et al., Kaon matrix elements and cp-violation from quenched lattice qcd. $i$ : The 3-flavor case, Phys. Rev. D68 (2003) 114506 [hep-1at/0110075].

[3] J. F. Donoghue, E. Golowich and B. R. Holstein, Dynamics of the standard model, Camb. Monogr. Part. Phys. Nucl. Phys. Cosmol. 2 (1992) 1-540.

[4] T. Blum et al., The $K \rightarrow(\pi \pi)_{I=2}$ Decay Amplitude from Lattice QCD, Phys. Rev. Lett. 108 (2012) 141601 [arXiv:1111.1699 [hep-lat]]. 
[5] T. Blum et al., $K \rightarrow \pi \pi \Delta I=3 / 2$ decay amplitude in the continuum limit, Phys. Rev. D91 (2015), no. 7074502 [arXiv:1502.00263 [hep-lat]].

[6] L. Lellouch and M. Luscher, Weak transition matrix elements from finite-volume correlation functions, Commun. Math. Phys. 219 (2001) 31-44 [hep-1at/0003023].

[7] C.-h. Kim and N. H. Christ, $K \longrightarrow$ pi pi decay amplitudes from the lattice, Nucl.Phys.Proc.Suppl. 119 (2003) 365-367 [arXiv:hep-1at/0210003 [hep-1at]].

[8] M. Luscher, Volume dependence of the energy spectrum in massive quantum field theories. 2. scattering states, Commun. Math. Phys. 105 (1986) 153-188.

[9] M. Luscher, Two particle states on a torus and their relation to the scattering matrix, Nucl. Phys. B354 (1991) 531-578.

[10] A. J. Buras, Kaon Theory News, 2015. arXiv:1510.00128 hep-ph].

[11] N. Ishizuka, K. I. Ishikawa, A. Ukawa and T. Yoshi, Calculation of $K \rightarrow \pi \pi$ decay amplitudes with improved Wilson fermion action in lattice $Q C D$, arXiv: 1505.05289 hep-lat]

[12] RBC, UKQCD Collaboration, N. H. Christ, T. Izubuchi, C. T. Sachrajda, A. Soni and J. Yu, Long distance contribution to the KL-KS mass difference, Phys. Rev. D88 (2013) 014508 \begin{tabular}{|l|l|l|l|l|}
\hline arXiv:1212.5931 & hep-lat].
\end{tabular}

[13] Z. Bai, N. H. Christ, T. Izubuchi, C. T. Sachrajda, A. Soni and J. Yu, $K_{L}-K_{S}$ Mass Difference from Lattice QCD, Phys. Rev. Lett. 113 (2014) 112003 [arXiv:1406.0916 $[$ hep-1at]].

[14] Z. Bai, $K_{L}-K_{S}$ mass difference computed with a 171 MeV pion mass, PoS LATTICE2014 (2015) 368 \begin{tabular}{|l|l|l|l|}
\hline arXiv:1411.3210 hep-lat]. &
\end{tabular}

[15] N. H. Christ, X. Feng, G. Martinelli and C. T. Sachrajda, Effects of finite volume on the KL-KS mass difference, Phys. Rev. D91 (2015), no. 11114510 [arXiv:1504.01170

[16] J. Brod and M. Gorbahn, Next-to-Next-to-Leading-Order Charm-Quark Contribution to the CP Violation Parameter $\varepsilon_{K}$ and $\Delta M_{K}$, Phys. Rev. Lett. 108 (2012) 121801 [arxiv: ]108.2036 [hep-ph].

[17] Z. Bai and N. Christ, Computing the long-distance contributions to $\varepsilon_{K}$, PoS LATTICE2015 (2015) 342.

[18] G. Isidori, G. Martinelli and P. Turchetti, Rare kaon decays on the lattice, Phys. Lett. B633 (2006) 75-83 [arXiv:hep-lat/0506026 hep-lat]].

[19] RBC, UKQCD Collaboration, N. H. Christ, X. Feng, A. Portelli and C. T. Sachrajda, Prospects for a lattice computation of rare kaon decay amplitudes: $K \rightarrow \pi \ell^{+} \ell^{-}$decays, Phys. Rev. D92 (2015), no. 9 094512 [arXiv:1507.03094 [hep-lat]].

[20] X. Feng, N. Christ, A. Portelli and C. Sachrajda, Long-distance contributions to the rare kaon decay $K^{+} \rightarrow \pi^{+} v \bar{v}$, PoS LATTICE2015 (2016) 341. 